\title{
Quality Guidelines for Systemic Lupus Erythematosus: Slow But Steady Progress
}

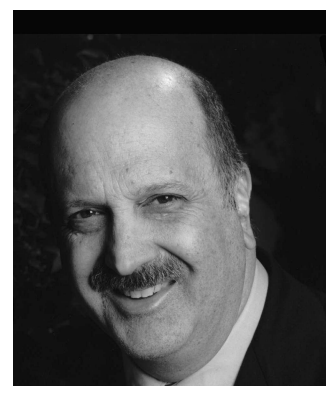

Systemic lupus erythematosus (SLE) places a significant burden on the US economy. It is estimated to be responsible for $\$ 10$ billion to $\$ 20$ billion in direct and indirect costs each year, costing the average patient with SLE an annual $\$ 10,000-\$ 20,000$ in accrued healthcare-related expenses ${ }^{1}$. These figures do not take into account the effects of loss of productivity and of disability, and that at least 10,000 people succumb directly to SLE annually (the psychological and societal effects of the disease).

In part because of the pleomorphic presentation of SLE and its many symptoms, signs, and laboratory abnormalities, it is difficult to make recommendations as to how often patients should be evaluated and monitored. In 1999, an American College of Rheumatology Ad Hoc Committee formulated guidelines for the management of SLE in adults $^{2}$. Although these eminence-based suggestions were prescient and well grounded, they are unfortunately now totally obsolete, in that they did not include targeted therapies, updated disease criteria, and newer insights garnered from biomarkers, imaging, clinical trials, and observational cohort studies.

In 2010, the European League Against Rheumatism (EULAR) made suggestions for monitoring patients with SLE in clinical practice and observational studies ${ }^{3}$. A total of 10 recommendations were presented based on Delphi surveys for prioritization, small group discussion, a systemic literature review considering levels of evidence, and nominal groups. The panel included input from rheumatology, internal medicine, dermatology, and nephrology. The recommendations are summarized as (1) patient assessment, emphasizing quality-of-life questionnaires; (2) consideration of cardiovascular risk factors; (3) screening for comorbidities (e.g., malignancy, osteoporosis); (4) consideration of infection risk; (5) frequency of assessments (every 6 to 12 mo); (6) laboratory assessments; (7) more stringent definitions of cutaneous lupus subsets; (8) use of measures that have predictive values for nephritis; (9) improved assessment of neuropsychiatric symptoms and signs; and (10) eye evaluations (e.g., antimalarial retinopathy, steroid risk for cataracts, glaucoma).

Although the conclusions of the group are generally valid, this writer found them to be diffuse, not interconnected or focused, and a bit hard to read and follow. For example, they fail to mention which tests should be obtained at what interval for monitoring corticosteroids, antimalarials, nonsteroidals, immune suppressives, or biologics. In other words, a health management organization would not be able to use this information.

Although Drs. Gladman and Urowitz were co-authors of the 1999 ACR guidelines, they did not participate in the EULAR initiative. Their lupus clinic in Toronto has followed patients prospectively since 1970 and has published hundreds of seminal observations and insights. Recently, these investigators and their colleagues focused upon how often asymptomatic patients should be followed ${ }^{4}$. In a prior survey, the Toronto group reviewed their experience with "serologically active, clinically quiescent" disease and found that $59 \%$ flared over a 3 -year period ${ }^{5}$. In a recent article, 515 asymptomatic patients presented for 3125 visits at an average of 3.8 month intervals. Over a 2 -year period, $25 \%$ had 1 of 10 "solitary silent variables" that were not recognized by patients as being suggestive of increased disease activity. Half of the variables were renal (proteinuria, hematuria, pyuria, casts, elevated serum creatinine), 3 hematologic (thrombocytopenia, leukopenia, low hemoglobin), and 2 were laboratory/serologic (anti-dsDNA and low complement). Half of recrudescences were renal, half were laboratory/serologic, and only $12 \%$ were hematologic. They concluded that patients with mild or inactive disease should be followed with clinical and laboratory measures at 3 to 4 month intervals. This specific information is important in guiding healthcare providers on what might be the "gold standard" for following SLE. This information comes with several caveats: it applies to a

See Frequency of visits in asymptomatic SLE, page 630

Personal non-commercial use only. The Journal of Rheumatology Copyright @ 2013 . All rights reserved. 
largely literate, compliant, and insured white Canadian population. It may not be relevant to all demographic, geographic, racial, or ethnic groupings. Not everybody can read or write, has access to an involved consultant 24/7, has transportation to the clinic, and can afford their medication. Further, although flares can be missed if patients are seen every 6-12 months (as in the EULAR publication), only $3-6 \%$ of the Toronto patients were started on a new steroid, antimalarial, or immune suppressive as a result of quarterly monitoring. How important are "solitary silent new features"? Important enough to demonstrate that one of the 10 EULAR recommendations is probably incorrect and misleading.

The passage and implementation of health reform in the United States relies heavily on cost-effectiveness. One of the principal components of US President Obama's reform initiative involves establishing Accountable Care Organizations (or ACO) ${ }^{6}$. It is widely believed (based on preliminary data from pilot sites) that integrating quality standards (e.g., judicious use of laboratory, imaging, consulting visits, physician education, and measurements of disease activity) into a non-coercive but evidence-based healthcare environment would save trillions of dollars while rewarding those who deliver exemplary medical care and without resorting to rationing. While standards and guidelines for approaching common conditions such as pneumonia, hypertension, diabetes, and congestive heart failure are slowly being implemented in selected settings, less common conditions such as SLE will lag behind. Yazdany, et al have started the ball rolling with the publication of a preliminary quality indicator set ${ }^{7}$. If the rheumatology community fails to act, a governmental organization will. The current situation for SLE is not satisfactory. No current guidelines take into account specialist access, demographics, children, the managed care/indigent environment, costs of medication, and use of available resources. It is also not clear how much of this burden should be shouldered by internal medicine, primary care, or rheumatology ${ }^{8}$. Who should manage hypertension, for example, in a patient with SLE, and how is this coordinated with their rheumatologic monitoring? OMERACT (Outcome Measures in Rheumatology Clinical Trials) develops guidelines for many rheumatic diseases, but has not had an SLE working group meeting since 1998 (a lapse in the process of being corrected). The publication of clinical trials for targeted therapies (e.g., belimumab, epratuzumab) has finally demonstrated that specific metrics for inflammation, damage, and quality of life have prognostic relevance ${ }^{9}$. For patients with SLE to be optimally managed, these insights should be integrated with the work from the Toronto group and with evidence-based suggestions of how to monitor SLE medications and disease activity through laboratory and other forms of clinical testing (e.g., imaging).

DANIEL J. WALLACE, MD, FACP, FACR, Associate Director, Rheumatology Fellowship Program Clinical Professor of Medicine,

Cedars-Sinai Medical Center,

David Geffen School of Medicine at UCLA, Los Angeles, CA, USA. E-mail: dwallace@ucla.edu

\section{REFERENCES}

1. Panopalis P, Clarke AE, Yelin E. The economic burden of systemic lupus erythematosus. Best Pract Res Clin Rheumatol 2012; 26:695-704.

2. American College of Rheumatology Ad Hoc Committee on Systemic Lupus Erythematosus Guidelines. Guidelines for referral and management of systemic lupus erythematosus in adults. Arthritis Rheum 1999;42:1785-96.

3. Mosca M, Tani C, Aringer M, Bombardieri S, Boumpas D, Brey R, et al. European League Against Rheumatism recommendations for monitoring patients with systemic lupus erythematosus in clinical practice and in observational studies. Ann Rheum Dis 2010;69:1269-74.

4. Gladman DD, Ibanez D, Ruiz I, Urowitz MB. Recommendations for frequency of visits to monitor systemic lupus erythematosus in asymptomatic patients: Data from an observational cohort study. J Rheumatol 2013;40:630-3.

5. Steiman AJ, Gladman DD, Ibañez D, Urowitz MB. Prolonged serologically active clinically quiescent systemic lupus erythematosus: frequency and outcome. J Rheumatol 2010;37:1822-7.

6. Press MJ, Michelow MD, Macphail LH. Care coordination in accountable care organizations: moving beyond structure and incentives. Am J Manag Care 2012;18:778-80.

7. Yazdany J, Panopalis P, Gillis JZ, Schmajuk G, MacLean CH, Wofsy D, et al, and Systemic Lupus Erythematosus Quality Indicators Project Expert Panels. A quality indicator set for systemic lupus erythematosus. Arthritis Rheum 2009;61:370-7.

8. Wallace DJ. Improving the prognosis of SLE without prescribing lupus drugs and the primary care paradox. Lupus 2008;17:91-2.

9. Navarra SV, Guzmán RM, Gallacher AE, Hall S, Levy RA, Jimenez RE, et al, and BLISS-52 Study Group. Efficacy and safety of belimumab in patients with active systemic lupus erythematosus: a randomized, placebo-controlled, phase 3 trial. Lancet 2011;377:721-31.

J Rheumatol 2013;40:542-3; doi:10.3899/jrheum.130128 